\title{
Design and implementation of VVOIP telephone based on Android
}

\author{
Xiangdong You ${ }^{1, a^{*}}$ \\ ${ }^{1}$ Beijing University of Posts \& Telecommunications, China \\ ayouxiangdong@bupt.edu.cn
}

\begin{abstract}
Keywords: Android VVOIP ARM SIP H264
Abstract. This paper proposes a design of VVOIP (Video and Voice over IP) on smart devices, with ARM Cortex A8 CPU structure in hardware, Android OS and JAVA language in software and C in Native layer. In the VVOIP, SIP is adopted as session protocol, and RTP packages carry H264 video streamer and GSM audio streamer.
\end{abstract}

\section{Introduction}

With rapid development of mobile Internet in recent years, smart devices based on mobile terminal change with each passing day. Among operating systems of intelligent terminals, IOS and Android OS account for a large proportion in the consumer electronics market. As traditional voice-only calls have been unable to meet consumers' needs, smart terminal based videophone has been accepted by more and more people, especially by households and enterprises.

It is not hard to understand that video calls should meet higher requirements than voice calls, such as more powerful CPU processing capacity and higher bandwidth. However, with the booming of semiconductor chip industry and the popularity of broadband Internet, both these two key issues can be overcome. The design of the VVOIP phone based on Android proposed in this paper can provide smoother video calls.

\section{The principles of VVOIP}

VVOIP is a high-performance network terminal on overall, using ARM Cortex A8 Processor. Soft-switch technology is adopted for the call part. Call process is achieved mainly by SIP and RTP protocols, which are responsible for the set-up of the call and the interaction of data during calls separately. Another feature of video phone is the acquisition, compression and transmission of multimedia data. We used H264 hard coding in video encoding part, while VP8, H264[1], MPEG4 soft coding is also supported.

SIP Session Initiation Protocol (SIP) was developed by the IETF in 1999. It is mainly used for the setting up, modifying and releasing of the session in network communication. In another word, SIP manages session by solving most of the problems in the setting-up of the session, simplifying the application layer software.[2] Just like HTTP, SIP is also a plain text protocol, which makes it possible to work among different systems. SIP belongs to the session layer in OSI, over the transport layer. SIP is widely used in multimedia communication, and it is much more popular now with the development of multimedia communication technologies.

Features of SIP protocol[2] include convenience, easy development, flexibility, suitability for various applications and compatibility with other technologies.

RTP People are sensitive to audio data in the multimedia communication. Both the loss of a data frame and a long delay will result in noise and will lead to decline in user experience; while for video data, the missing of few frames or a delay will only cause the image mosaic, which does not have much influence in user experience. From this we can see that audio and video has different requirements for network transmission in multimedia communications. There are two transmission modes in IP network, one is the connection-oriented TCP, and the other is the connectionless UDP. UDP cannot 
guarantee the reliability of the audio data, while TCP's three-way handshake will cause unnecessary delay. What's more, TCP can't realize retransmission.

Through this analysis we can find that the original transport-layer protocol cannot support multimedia data transmission. Out of this demand, the International Organization for Standardization has developed a set of RTP/RTCP Protocol for transmission of multimedia data streams. .

Video coding technique $\mathrm{H} 264$ is a new video compression standard developed by a joint video team which consists of ISO/IE and ITU-T. H264 has three subsets: basic subsets, subject subsets and extensions. Basic subset was designed specifically for video conferencing applications, which provides powerful error cancellation technique and supports low-latency encoding/decoding technology making the display of video conferences much more natural.

H264 puts forward two new concepts: Video Coding Layer (VLC) and Network Adapter Layer (NAL). The former is a description of the compression of video content information, which defines the syntax of the macro block layer and layers below; while the latter is a description of transmission through specific network, which defines the syntax of the slice layer and layers above. This structure makes information encapsulation much easier and provides better priority control of information to adapt to different network applications. By adopting H264 video coding standard for network transmission, high compression ratio and stronger robustness can be achieved. According to RFC3984, there are three types of subcontracting modes when using RTP to carry H264's NAL unit: Single NAL unit mode, Interleaved mode and Non-interleaved mode. [4]

All forms of the packets of data extraction units in $\mathrm{H} 264$ are called simple package. This packet strategy is to head a NAL packet into a RTP packet before sending it. H264 video streams should be packaged into RTP packets following the RTP specification when transporting over RTP. According to the encapsulation of RTP specification [5], and to avoid data segmentation in IP layer, NAL units generated in video encoding procession should not be larger than the maximum size of transmission unit.

\section{Hardware design of VVOIP}

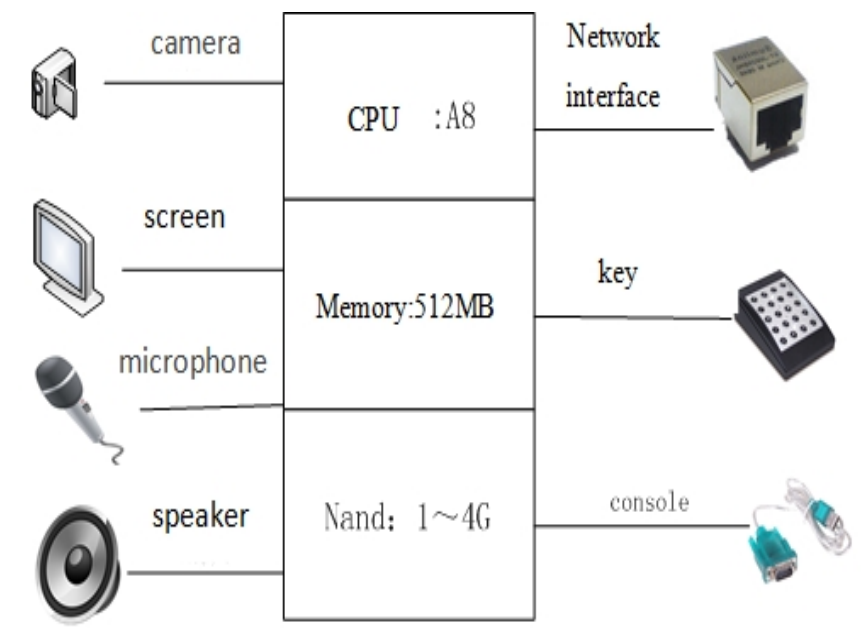

Fig.1 Hardware frame of VVOIP

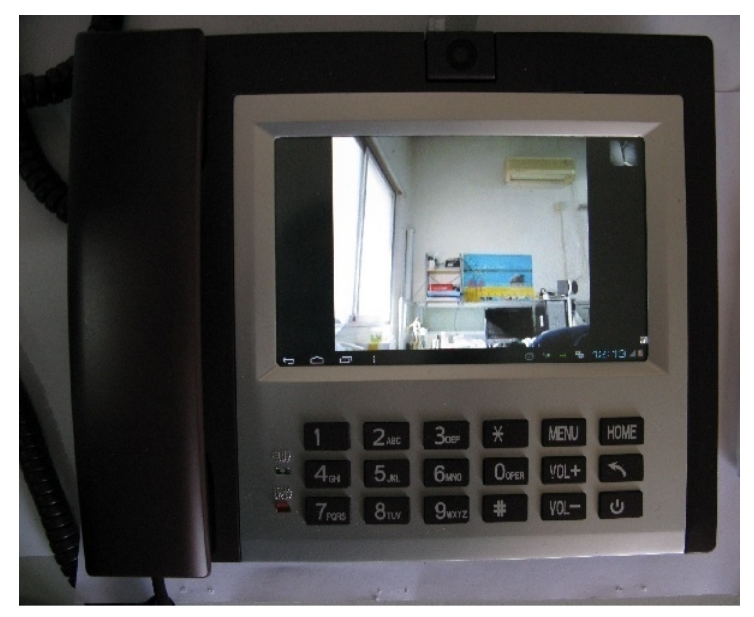

Fig. 2 Prototype of VVOIP

Hardware diagram of VVOIP Its core circuit adopts S5PV210 produced by Samsung as core processors. This processor was developed based on ARM Cortex-A8 processors, and its clock can speed up to $1 \mathrm{GHz}$. S5PV210 contains an integrated graphics engine called PowerVR SGX540. This VVOIP was configured by a memory of $512 \mathrm{MB}$ and a FLASH memory of $2 \mathrm{~GB}$, and its function module contains a network interface, a keyboard, CONSOLE interfaces, digital cameras, LCD monitors, microphones, speakers and so on, as shown in Figure 1.

Interfaces of VVOIP Different from the traditional telephone, smart video phone based on Android has a more complex structure, so as to cover more function modules and provide various interfaces to meet more demands. In addition to the basic handle interfaces, it should also provide interfaces to 
Ethernet, USB, LCD and so on. Based on the functional and performance requirements of VVOIP, we designed more than one telephone interfaces as shown in table 1.

\begin{tabular}{|c|c|c|c|}
\hline Interface & Performance & Number & Description \\
\hline Wired networkRJ45 & 10/100Mbps & 1 & One RJ45 socket \\
\hline Camera & CMOS camera & 1 & VGA or above \\
\hline Monitor & Seven inches TFT LCD & 1 & $800 \times 480$ or above \\
\hline Key & Basic functional keys for Android & 5 & $\begin{array}{l}\text { One switch/standby key and } \\
\text { five common key named } \\
\text { K1-K5 }\end{array}$ \\
\hline USB HOST & Host USB 2.0 & 1 & One USB host socket \\
\hline USB OTG & OTG USB 2.0 & 1 & Used for ADB for Android \\
\hline LEDs & $\begin{array}{l}\text { Red LED for power } \\
\text { Green LED for network }\end{array}$ & $1-4$ & $\begin{array}{l}\text { LED for network is put on } \\
\text { RJ45 socket }\end{array}$ \\
\hline SD card socket & SD card & 1 & Mini SD-card socket \\
\hline Phone handle & $\begin{array}{l}\text { Including one speaker and one } \\
\text { MIC }\end{array}$ & 1 & four core handle socket \\
\hline $\begin{array}{l}\text { Hands-free } \\
\text { telephone circuit }\end{array}$ & $\begin{array}{l}\text { One Speaker of } 8 \Omega \text { and } 1 \mathrm{~W} \text {, one } \\
\text { MIC }\end{array}$ & 1 & Speaker( $8 \Omega 1 \mathrm{~W})$ \\
\hline power & $5 \mathrm{~V} / 2 \mathrm{~A}$ & 1 & $220 \mathrm{~V} / 5 \mathrm{~V}$ power adapter \\
\hline $\begin{array}{l}\text { Insert spring of } \\
\text { handle }\end{array}$ & Pick-up machine testing & 1 & $\begin{array}{l}\text { Provide pick-up machine } \\
\text { detection ting handles }\end{array}$ \\
\hline
\end{tabular}

Table 1: Interfaces of VVOIP

\section{Software design of VVOIP}

Linphone Linphone is an open source VoIP software supported by many platforms such as Android 、 ISO 、 Linux and so on. Linphone uses hierarchical design so that the programmers can transform it as they want based on their system characteristics to develop customized products. Linphone uses SIP protocol as the call signaling, and transports media streaming via RTP/RTCP, which support the communication among different software using the same standard protocol. As for the design proposed in this passage, it is a must to reform Linphone in order to improve the efficiency of the telephone. The reformation, such as replacement of hardware and software encoding, will promote the operating efficiency of the entire system.

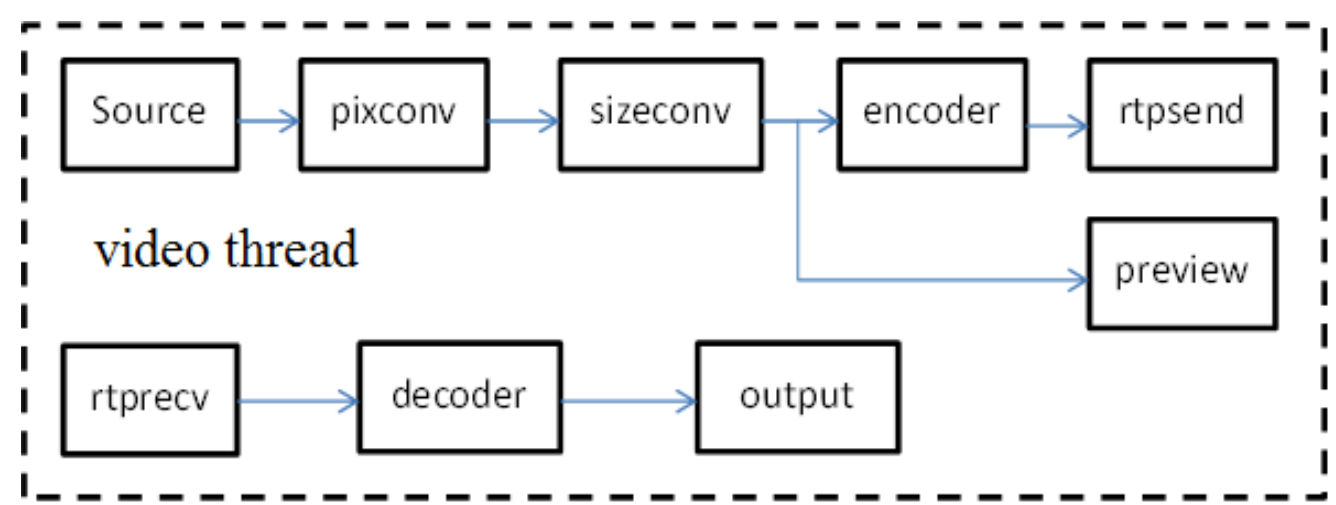

Figure 3: Mediastreamer2 video thread

Linphone adopts Mediastreamer2 as the multimedia processing module, which is the core module of Linphone's streaming media handling module. The capturing of audio and video massages, the sending and receiving of RTP streaming of audio and video massages, the encoding and decoding of audio and video massages and the playing of audio and video massages are all accomplished in this module. Its core work is completed by three threads: video stream processing thread (Figure 3), audio processing thread as well as video capturing thread. Filter structure is the core interface of Mediatreamer2. A 
Filter object is a process, and a complete streaming media process will be built up after connecting the different Filters. Filters transport data directly through a data queue.

Transplantation and transformation of Linphone software Compilation of Linphone library. In order to achieve better performance of $\mathrm{H} 264$ hardware encoding, you need to call a third party library file and to replace the original API of H264 by a new API which supports hardware encoding. On Android platform, H264 of Linphone is a separate module. In order to obtain better results, an overall optimization should be given to this part. First of all, $d$ a referenced third-party library should be added in the Android.mk file. Compile the file by using NDK(Native Debug Kit) on Android platform, after getting the new H264 library file that supports hardware encoding. Don't forget to place the new library file to the place specified by the system to make the new library file accessible for applications of upper layer, and then hardware video encoding can be realized.

\section{Prototype of VVOIP}

As is shown in figure 2, this VVOIP adopts H.264 for video processing and SPEEX coding standard for audio processing during two-way communication. This phone works well, and indicators of the prototype are listed as follows:

Video encoding/decoding: VP8, H.264 and MPEG4 supported

Video resolution: QCIF, CIF and VGA

Frame rates: no less than $15 \mathrm{fps}$, adaptable according to bandwidth

Video bit-rate: 280-330kbps (CIF, 15fps)

Audio encoding/decoding: G.711u, G.711a, G929, GSM and SPEEX supported

Network interfaces: 10M/100Mbps cable RJ45, 802.11g Wireless and WiFi

\section{Conclusion}

This paper proposed the design and implementation of a visual VOIP based on Android and ARM Cortex-A8, which uses SIP protocol as call signaling. This telephone supports registration, call and video call, and adopted RTP Protocol to transmit call media streaming. Among this, its audio encoding supports G711A, G711U, G722, GSM, SPEEX 8K and SPEEX 16K, and its video encoding supports H264, VP8 and MPEG4.

The design ideas of the VVOIP proposed in this paper can be used in in-vehicle wireless video monitoring, network monitoring and many other fields. In a word, it can be widely promoted, and has tremendous commercial value in the internet of things in the future.

\section{References}

[1] Yoursef O. Sharrab and Nabil J. Sarhan, Detailed Comparative Analysis of VP8 and H.264, 2012 IEEE Symposium on Multimedia, pp. 133 - 140, 2012.

[2] Zhang Youbo, Zhang Huanqiang and Sun Liming, Design and implementation of video conference system based on SIP, Computer Engineering,Vol 31, Nov 2005 In Chinese

[3] Zhang Haijun, Zhang Jianjun, Yang Yingeng and Wu Kejie, Design and implementation of RTP transmission control and real-time video monitoring system, Microcomputer and Application vol 28

( 5 ) , 2009. In Chinese

[4] Li Xin, Network video transmission based on H.264, Beijing University of Posts and Telecommunications, 2006 In Chinese

[5] Schulzrinne H. Casner S. RTP: A Transport Protocol for Real-Time Application, RFC3550, 2003. 